schwefelsaures Eisenoxyd nimmt, wovon ich mich durch Versuche überzeugte. In allen Fällen ist die Lösung stark alkalisch; auch kann man sowohl Kalilauge, als auch Wasser in beliebiger Quantität hinzusetzen, ohne Eisenoxyd zu fällen. Dies Verhalten ist also vorläufig zu beruicksichtigen; ausgedehntere Betrachtungen hierüber bleiben der Zukunft vorbehalten.

\title{
Zweiter Aufsatz über Saturationen;
}

von

Dr. d u M ên il.

Es thut mir leid, dem Herrn Dr. Mohr durch meine Gründe gegen die extemporirten Saturationen missfällig gewesen zu sein; dieses war keineswegs mein Wille, sondern nur dadurch nützen wollte ich.

Sehr erinnerlich ist es mir, dass die Ansichten des Dr. Mohr schon vor vielen Jahren in irgend einer Zeitschrift, und zwar unter den seinigen ganz ähnlichen Phrasen zur Sprache kamen; auch damals missbilligte ich sie nach reifer Erwägung und Erfahrung, und bin noch jetzt völlig überzeugt, die neuere Methode, Potio Riverii zu bereiten - die der Herr Doctor einen alten Schlendrian nennt - sei eine wahre Verbesserung.'

Meine fruheren Worte gegen die Saturationen passen noch so gut auf den zweiten Aufsalz des erwähnten Herrn Verfassers, dass sie hier wiederholt werden müssten; doch soll es nicht geschehen, ich will demselben nur hinzufügen, es könne die wenige Kohlensäure einiger Löffel voll der Mohr'schen Potio Riverii nicht sein, welche die Heilung eines krankhaften Erbrechens bewirkt; denn ich sah Anfälle dieses Uebels auch mit unserm Präparate gehoben. Es scheint mir, dass die ganze Mischung desselben, also auch derdurch Erwärmung nicht entfernt werdende, gleichsam balsamische Geruch des Essigs sie mit hervorbringt. Schliesslich gedenke ich noch, dass sich uber die in 
Kraemer, zur Kenntniss flüchiger Pflanzensäuren. 9

beiden Aufsätzen des besagten Herrn Verfassers geäusserten Meinungen und Behauptungen Manches einwenden liesse, wenn man es der Mühe werth halten könnte, uber eine Blase von Kohlensäuregas - um welche sich doch eigentlich die ganze Sache dreht und die der Herr Dr. Mohr doch wohl nur allein von grosser Wichtigkeit oder mit homöopathischer Phantasie für ein Specificum hält, zu disputiren.

Doch noch eins; ich nannte den Herrn Dr. Mo hr einen verdienten und würdigen Mann - was er auch bis jetzt in meinen Augen noch ist - er mich dafür einen gefeierten. Da dieser Ausdruck hier aber mehrdeutig erscheint, so erkennen ihn Viele für einen Druckfehler und lesen: gefeuerten. In der That mögen sie Recht haben, denn der Herr Doctor feuerte gewaltig auf mich los, doch ohne eine Verwundung, sondern bloss eine Verwunderung zu verursachen $*$.

\section{Zur Kenntniss einiger flïclitigen Pflanzensäuren;}

von

H. I raemer in Firchen.

Die in dieser Zeitschrift LI. 1. enthaltene Abhandlung des Herrn Geh. Ober-Berg-Commissairs Dr. du Mènil über Wermuth- und Lavendelsäure giebt mir Veranlassung, einige Versuche zu erwähnen, die ich in Betreff mehrerer in Begleitung flüchtiger Oele auftretenden Pflanzensäuren gemacht habe, und die, wenn sie auch zu einem sichern Resultate nicht gefuhrt haben, für die Kenntniss dieser Säuren nicht ohne Interesse sein mögen.

Redtenbacher's interessante Forschungen über die Oxydationsproducte verschiedener Fettarten begründen die

*) Vom pharmaceutischen und chemischen Standpuncte aus däucht uns diese Angelegenheit nun genug besprochen. Wir überlassen daher von nun an die weitere Entscheidung der praktischen Medicin.

Die Red. 